\title{
Simulation of Top Quark Production for the ATLAS Experiment
}

Ian Connelly*, on behalf of the ATLAS Collaboration

Royal Holloway, University of London

E-mail: ian.connelly@ern.ch

The Monte Carlo setups used by ATLAS to model the $t \bar{t}$ and single-top production in $13 \mathrm{TeV}$ pp collisions are described. The performance of different event generators is assessed by comparing measurements at $7 \mathrm{TeV}, 8 \mathrm{TeV}$ and $13 \mathrm{TeV}$ to predictions from simulated data. The evaluation of systematic uncertainties and the dependence of generator predictions on the tuning parameters are also discussed.

38th International Conference on High Energy Physics 3-10 August 2016

Chicago, USA

${ }^{*}$ Speaker. 


\section{Introduction}

Successful measurements and searches by the ATLAS experiment [1] rely on accurate models and predictions of high energy particle collisions. Monte Carlo event generators are used to simulate a hard-scatter process (typically between quarks and/or gluons) which will result in a specific final state. A range of event generators are used to model different physics processes and the focus of the work presented at this conference was to study the setups used to model $t \bar{t}$ and single-top production in $13 \mathrm{TeV}$ proton-proton collisions [2].

\section{Event Generators and Parton Showers}

Event generators use hard-scattering matrix elements (ME) to calculate cross-sections up to a fixed-order. Parton showers (PS) model the fragmentation and hadronisation of partons. Generators can differ on the procedure used for calculating real and virtual amplitudes and in the matching of next-to-leading order (NLO) radiation between ME and PS in order to preserve the total crosssection.

A range of samples are available for use in ATLAS analyses which use various combinations of event generators and parton showers. A summary of the samples is shown in Table 1 and further details on the setup with associated references can be found in [2-4]. The nominal $t \bar{t}$ and single-top samples used in ATLAS analyses are generated with POWHEG+PYTHIA6. An alternative sample generated with MG5_aMC@NLO provides a systematic uncertainty by varying the NLO matching scheme between PowHEg and MC@NLO. An alternative sample showered with HERwIG provides an additional systematic uncertainty by varying the hadronisation model.

\begin{tabular}{|c|c|c|c|c|}
\hline Event Generator & Parton Shower & Accuracy & ME PDF & Scale \\
\hline POWHEG & PYTHIA6 & NLO & CT10 & $\sqrt{m_{t}^{2}+p_{T, t}^{2}}$ \\
\hline POWHEG & PYTHIA8 & NLO & NNPDF3.0 & $\sqrt{m_{t}^{2}+p_{T, t}^{2}}$ \\
\hline POWHEG & HERWIG+ & $\mathrm{NLO}$ & CT10 & $\sqrt{m_{t}^{2}+p_{T, t}^{2}}$ \\
\hline POWHEG & HERWIG7 & $\mathrm{NLO}$ & CT10 & $\sqrt{m_{t}^{2}+p_{T, t}^{2}}$ \\
\hline MG5_aMC@NLO & PYTHIA8 & NLO & NNPDF3.0 & $H_{T} / 2$ \\
\hline MG5_aMC@NLO & HERWIG++ & $\mathrm{NLO}$ & CT10 & $\sqrt{m_{t}^{2}+\left(p_{T, t}^{2}+p_{T, \bar{t}}^{2}\right) / 2}$ \\
\hline MG5_aMC@NLO & HERWIG7 & $\mathrm{NLO}$ & CT10 & $\sqrt{m_{t}^{2}+\left(p_{T, t}^{2}+p_{T, \bar{t}}^{2}\right) / 2}$ \\
\hline SHERPA 2.2 & SHERPA & Multi-Leg NLO ${ }^{1}$ & NNPDF3.0 & $\sqrt{m_{t}^{2}+\left(p_{T, t}^{2}+p_{T, \bar{t}}^{2}\right) / 2}$ \\
\hline MADGRAPH & PYTHIA8 & Multi-Leg LO² & NNPDF3.0 & $\sqrt{\sum m_{T}^{2}} \forall$ partons \\
\hline
\end{tabular}

Table 1: Summary of $t \bar{t}$ samples available in ATLAS.

\footnotetext{
${ }^{1}$ SHERPA generates $t \bar{t}$ with up to 1 additional parton at NLO accuracy and up to 4 additional partons at LO accuracy.

${ }^{2}$ MADGRAPH generates $t \bar{t}$ with up to two additional partons at LO accuracy.
} 


\subsection{Powheg+Pythia8}

For MC used by the ATLAS experiment, a transition has occured moving from PYTHIA6 to PyTHIA8. This setup has been studied during the past year to improve the modelling of data by optimising the matching parameters in PYTHIA8. One topic which has an important consideration is the prescription for evaluating systematic uncertainties associated to varying the amount of QCD radiation in an event. A set of uncertainties have been determined for the A14 tuned set of parameters for the parton shower and underlying event [5]. These variations have been compared to $7 \mathrm{TeV}$ measurements to establish the impact on top physics. Variation 3c which changes $\alpha_{s}$ in the eigentune by $\pm 10 \%$ has been shown to have the largest impact and covers the size of the alternative variations. The coverage of this uncertainty is shown in Figure 1. Work is still ongoing to establish how to combine this uncertainty with scale variations to best cover measurements of additional radiation in $t \bar{t}$ events at $13 \mathrm{TeV}$.

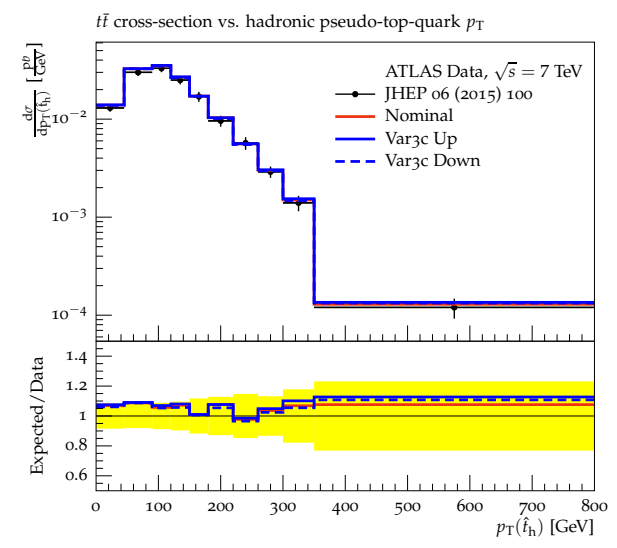

(a)

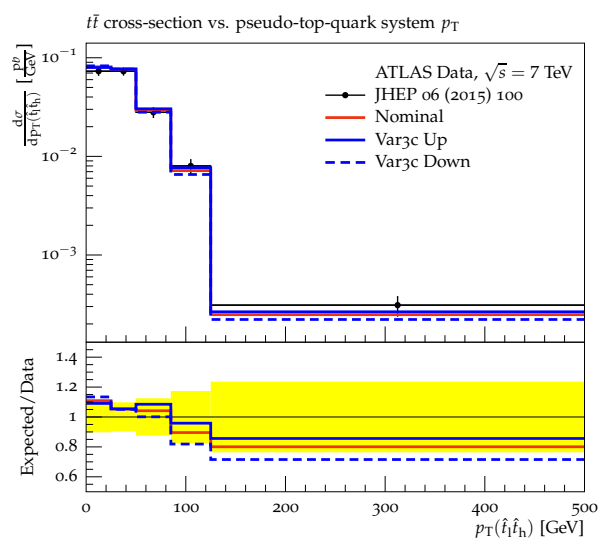

(b)

Figure 1: Comparison of A14 eigentune variation $3 \mathrm{c}$ as a function of the transverse momentum of the pseudo-top-quark (1a) and pseudo-top-quark pair system (1b) [3].

\subsection{HERWIG7}

The newest version of Herwig, Herwig7 [6], has been implemented in the ATLAS MC framework and has been tested when interfaced to PowHEG and to MG5_aMC@NLO for $t \bar{t}$ events. Some features of the HERWIG+ shower can be seen in Figure 2a where a slope in the leading jet $p_{\mathrm{T}}$ can be observed which does not agree within the uncertainties of the $7 \mathrm{TeV}$ measurements. The use of HERWIG7 is seen to improve this distribution, but some shortcomings in the underlying event tune have lead to excessive jet activity which can be observed in Figure $2 b$ and is being studied.

\subsection{Multi-Leg Generators}

Some analyses probe regions of extreme $t \bar{t}$ phase-space. In these cases, it is sometimes more effective to make use of multi-leg event generators. These samples generate $t \bar{t}$ with some given accuracy (LO or NLO) but then simulate an additional number of partons which have to be correctly 


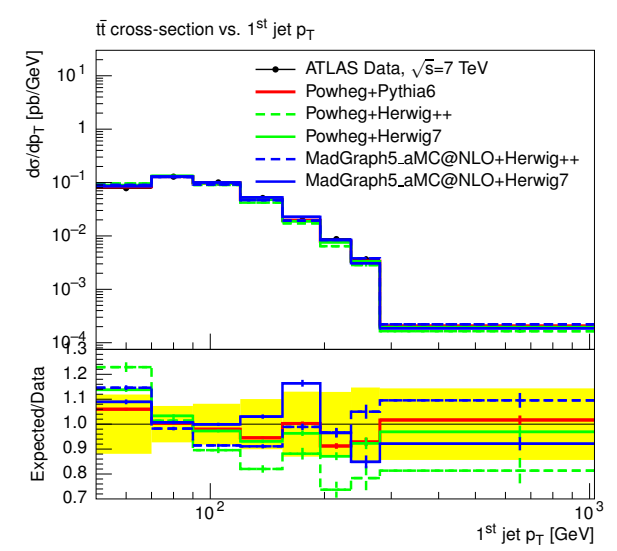

(a)

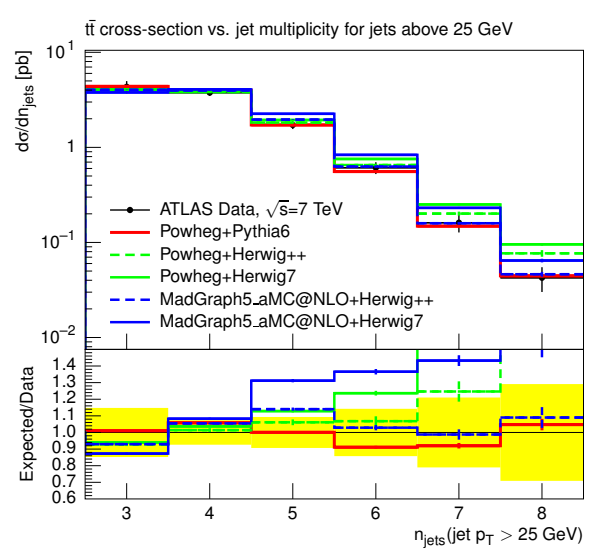

(b)

Figure 2: Comparison of HERWIG7 with other generators compared to $\sqrt{s}=7 \mathrm{TeV}$ measurements of leading jet $p_{\mathrm{T}}(2 \mathrm{a})$ and number of jets (2b) in $t \bar{t}$ events [2].

merged to prevent double-counting. These setups allow the matrix element generator to simulate additional radiation, rather than being dependent on the approximations applied by parton showers. There are three multi-leg samples in ATLAS which are being studied and used; a MADGRAPH sample which generates $t \bar{t}$ plus up to two additional partons at LO using the CKKW-L merging scheme [7,8], a MG5_aMC@ NLO sample which generates $t \bar{t}$ plus up to two additonal partons at NLO using the FxFx merging scheme [9], and a SHERPA sample which generates $t \bar{t}$ plus up to one additional parton at NLO and up to four further partons at LO using the MEPS@ NLO scheme [10].

\section{Summary}

A range of MC samples which model $t \bar{t}$ production are available in ATLAS. A comparison of NLO samples are shown in Figure 3 as a function of the transverse momentum of a hadronic top quark at $13 \mathrm{TeV}$.

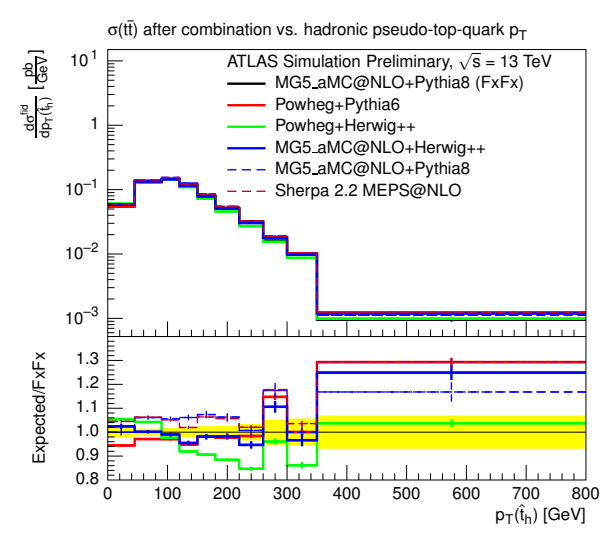

Figure 3: Summary of NLO $t \bar{t}$ MC samples available in ATLAS. The MC have been produced at $\sqrt{s}=13 \mathrm{TeV}$ and the ratio is shown with respect to the FxFx sample [2]. 


\section{References}

[1] ATLAS Collaboration, The ATLAS Experiment at the CERN Large Hadron Collider, JINST 3 (2008) S08003.

[2] ATLAS Collaboration, Further studies on simulation of top-quark production for the ATLAS experiment at $\sqrt{s}=13 \mathrm{TeV}$, Tech. Rep. ATL-PHYS-PUB-2016-016, CERN, Geneva, Aug, 2016.

[3] ATLAS Collaboration, Simulation of top quark production for the ATLAS experiment at $\sqrt{s}=13 \mathrm{TeV}$, Tech. Rep. ATL-PHYS-PUB-2016-004, CERN, Geneva, Jan, 2016.

[4] ATLAS Collaboration, Studies on top-quark Monte Carlo modelling for Top2016, Tech. Rep. ATL-PHYS-PUB-2016-020, CERN, Geneva, Sep, 2016.

[5] ATLAS Collaboration, ATLAS Run 1 Pythia8 tunes, Tech. Rep. ATL-PHYS-PUB-2014-021, CERN, Geneva, 2011.

[6] J. Bellm et al., Herwig 7.0/Herwig++ 3.0 release note, Eur. Phys. J. C76 (2016), no. 4 196, [arXiv:1512.0117].

[7] L. Lonnblad, Correcting the color dipole cascade model with fixed order matrix elements, JHEP 05 (2002) 046, [hep-ph / 0112284$].$

[8] L. Lonnblad and S. Prestel, Matching Tree-Level Matrix Elements with Interleaved Showers, JHEP 03 (2012) 019, [arXiv:1109.4829].

[9] R. Frederix and S. Frixione, Merging meets matching in MC@NLO, JHEP 12 (2012) 061, [arXiv:1209.6215].

[10] S. Höche, F. Krauss, M. Schönherr, and F. Siegert, QCD matrix elements + parton showers: The NLO case, JHEP 04 (2013) 027, [arXiv:1207.5030]. 